\title{
CISTOS E TUMORES ODONTOGÊNICOS: ESTUDO RETROSPECTIVO DOS CASOS DIAGNOSTICADOS NO PERÍODO DE 10 ANOS EM UM HOSPITAL NO SUL DO BRASIL
}

\author{
Odontogenic cysts and tumors: a retrospective study of the cases \\ diagnosed in a period of 10 years in a southern Brazilian hospital
}

\author{
(D)Bruna Luísa Neumann ${ }^{a}$, (DStefánie Thiemea, (D)Amanda de Farias Gabriela, \\ DVirgilio Gonzales Zanella ${ }^{a, b}$, (DFelipe Martins Silveirac, (D)Ricardo Gallicchio Kroef ${ }^{b}$, \\ (D) Marinez Bizarro Barrab, (D) Marco Antonio Trevizani Martins ${ }^{\mathrm{a}, \mathrm{d}}$, (D) Manoela Domingues Martins $s^{\mathrm{a}, \mathrm{b}}$
}

\section{RESUMO}

Introdução: as lesões odontogênicas (LOs) compreendem um grupo heterogêneo de patologias orais e maxilofaciais que apresentam características distintas. O objetivo do presente estudo foi identificar as características clínico-patológicas das LOs diagnosticadas em um hospital da região sul do Brasil. Materiais e métodos: foi realizado um estudo retrospectivo para levantamento dos casos com diagnóstico histopatológico de LOs no período entre 2007 e 2017. Os laudos dos pacientes foram avaliados para extração das características clínico-patológicas e dos diagnósticos histopatológicos de cada caso. Resultados: um total de 255 casos de LOs foram identificados. Destes casos, 197 (77\%) cistos odontogênicos e 58 (23\%) tumores odontogênicos foram coletados, sendo que somente um caso (0,39\%) possuiu o diagnóstico de neoplasia odontogênica maligna. Os diagnósticos mais prevalentes foram cisto radicular $(32,5 \%)$ e cisto dentígero (31,76\%), seguidos de ceratocisto odontogênico (10,98\%), odontoma (10\%) e ameloblastoma (10\%). A maioria dos casos acometeu mandíbula (53,7\%), com uma discreta predileção pelo sexo feminino (51\%). A média de idade foi de $34 \pm 20,53$ anos. Discussão: os dados apresentados corroboram com a literatura no que se refere à raridade do diagnóstico de tumores odontogênicos. Conclusão: o presente estudo demonstrou as principais características clínico-patológicas de LOs diagnosticadas em um hospital no sul do Brasil, contribuindo para um maior conhecimento do perfil destas lesões.
Palavras-chave: Cistos odontogênicos. Tumores odontogênicos. Patologia bucal. Diagnóstico bucal.

\section{ABSTRACT}

Introduction: odontogenic lesions (OLs) represent a heterogeneous group of oral and maxillofacial pathologies presenting distinct characteristics. The present study aimed to identify the clinical and pathological characteristics of OLs diagnosed in a southern Brazilian hospital. Materials and methods: a retrospective study was performed to evaluate cases with histopathological diagnosis of OLs identified in the period between 2007 and 2017. The patient's medical records were evaluated in order to obtain the clinical and pathological characteristics and the histopathological diagnosis from each case. Results: a total of 255 cases of OLs were identified. From these, 197 (77\%) odontogenic cysts and 58 (23\%) odontogenic tumors were surveyed, with only one case $(0,39 \%)$ of a malignant odontogenic neoplasm. The most prevalent diagnosis were radicular cyst (32.5\%) and dentigerous cyst (31.76\%), followed by odontogenic keratocyst (10.98\%), odontoma (10\%) and ameloblastoma (10\%). The majority of the cases affected the mandible (53.7\%) with a slight preference by female individuals (51\%). The mean age was $34 \pm 20.53$ years old. Discussion: the presented data are in accordance with the literature regarding the rarity of the diagnosis of odontogenic tumors. Conclusion: the present study demonstrated the main

\footnotetext{
aDepartamento de Patologia Oral, Faculdade de Odontologia, Universidade Federal do Rio Grande do Sul, Porto Alegre, RS, Brasil. bDepartamento de Cirurgia de Cabeça e Pescoço, Hospital Santa Rita, Complexo Hospitalar Santa Casa, Porto Alegre, RS, Brasil. ${ }^{c}$ Departamento de Diagnóstico Oral, Faculdade de Odontologia de Piracicaba, Universidade Estadual de Campinas, Piracicaba, SP, Brasil. dDepartamento de Medicina Oral, Hospital de Clínicas de Porto Alegre, Porto Alegre, RS, Brasil.
}

Autora de correspondência: Manoela Domingues Martins - E-mail: manomartins@gmail.com Data de envio: $18 / 10 / 2020$ | Data de aceite: $11 / 01 / 2021$ 
clinical and pathological characteristics of OLs diagnosed in a southern Brazilian hospital, contributing to a better understanding of these injuries profiles.

Keywords: Odontogenic cysts. Odontogenic tumors. Pathology, oral. Diagnosis, oral.

\section{INTRODUÇÃO}

As lesões odontogênicas (LOs) constituem um grupo heterogêneo de patologias orais e maxilofaciais ${ }^{1}$. Essas lesões são originárias dos remanescentes teciduais epiteliais e/ou mesenquimais do desenvolvimento dentário, tendo como exemplos os restos epiteliais de Malassez, a lâmina dentária e o órgão do esmalte ${ }^{2-4}$. As LOs, que compreendem os cistos odontogênicos (COs) e os tumores odontogênicos (TOs), são entidades incomuns que apresentam dados epidemiológicos variáveis. Relata-se que menos de $2-3 \%$ de todas as amostras enviadas para análise histopatológica nos serviços de patologia oral representam algum tipo de $\mathrm{LO}^{5}$. Os COs são etiopatologicamente classificados como cistos inflamatórios, cujos fatores etiológicos provêm da inflamação, ou como cistos de desenvolvimento, cujos fatores desencadeantes ainda são desconhecidos ${ }^{6,7}$.

Os TOs podem variar desde proliferações hamartomatosas até neoplasias benignas ou malignas com potencial metastático ${ }^{8,9}$. Seu desenvolvimento pode ocorrer centralmente, no interior do osso, ou perifericamente, em regiões extra ósseas, e sua etiopatogenia ainda é incerta ${ }^{9-11}$. Os TOs são lesões relativamente raras, compreendendo cerca de $1 \%$ de todos os tumores que se desenvolvem nos ossos gnáticos ${ }^{12-14}$. Estas neoplasias apresentam diferentes aspectos clínicos e histopatológicos podendo variar desde uma apresentação clínica com crescimento assintomático, lento e expansivo até casos com desenvolvimento rápido, infiltrativo e sintomático ${ }^{2,15,16}$. A mais recente classificação da Organização Mundial da Saúde (OMS) passou a categorizar os TOs em epiteliais, mesenquimais e mistos, representando uma versão mais simplificada quando em comparação às anteriores ${ }^{17}$.

A definição das características clínico-patológicas das LOs é essencial para o conhecimento e o estabelecimento de suas principais características. Estudos anteriores demonstram diferenças epidemiológicas em relação a COs e TOs de acordo com a região em que são analisados. Entretanto, até onde se sabe, não existem estudos avaliando as distintas características clínico-patológicas de LOs diagnosticadas em um hospital sul brasileiro referência em oncologia. Nesse contexto, o objetivo do presente estudo foi identificar as características clínico-patológicas das LOs diagnosticadas em um hospital localizado no sul do Brasil.

\section{MATERIAIS E MÉTODOS}

\section{Seleção da amostra}

Após a aprovação pelo Comitê de Ética em Pesquisa (n. ${ }^{\circ}$ CAAE 76809417.2.0000.5335), os casos de interesse foram levantados no sistema eletrônico hospitalar de Anatomia Patológica da Irmandade da Santa Casa de Misericórdia de Porto Alegre. Foram incluídos todos os casos resultantes do cruzamento da topografia (região de cabeça e pescoço) com o diagnóstico histopatológico (COs e TOs), no período compreendido entre 2007 e 2017. Dentre os critérios de exclusão estavam: (1) diagnóstico histopatológico indefinido ou compatível com lesão não-odontogênica e (2) cadastros repetidos do mesmo paciente. 


\section{Coleta de dados}

Cada caso foi avaliado por pesquisadores previamente calibrados e foram registradas as informações dos laudos em um formulário de coleta de dados contendo: (1) diagnóstico histopatológico por tipo de LO, (2) dados demográficos (sexo e idade) e (3) localização anatômica. Os diagnósticos selecionados foram classificados de acordo com a atual proposta da OMS/2017. Foi criado um banco de dados com as informações coletadas nos laudos e relacionadas à análise morfológica das lesões.

\section{Análises estatísticas}

Foi realizada uma análise estatística descritiva calculando média, desvio padrão, máximo, mínimo e mediana para variáveis quantitativas e frequências e porcentagens para variáveis qualitativas.

\section{RESULTADOS}

Inicialmente, foram levantados um total de 370 casos a partir da pesquisa por localização anatômica associada ao diagnóstico histopatológico. Destes, 115 foram excluídos pelos seguintes motivos: diagnóstico indefinido e/ou lesão não-odontogênica $(\mathrm{n}=10)$; estruturas do desenvolvimento dentário não-patológicas $(n=25)$; e casos repetidos $(n=80)$. Com isso, 255 casos de LOs representaram a amostra final, dos quais 197 casos (77\%) possuíam o diagnóstico de COs e $57(22,61 \%)$ de TOs benignos. Somente um caso $(0,39 \%)$ representou um tipo de neoplasia odontogênica maligna, como descrito na Tabela 1.

Os diagnósticos mais prevalentes foram cisto radicular $(\mathrm{n}=83,32,5 \%)$, cisto dentígero $(n=81,31,76 \%)$, ceratocisto odontogênico $(n=28,10,98 \%)$, odontoma $(n=26,10 \%)$ e ameloblastoma $(\mathrm{n}=25,10 \%)$. Os outros diagnósticos corresponderam a um total de 12 casos $(4,91 \%)$, incluindo cisto odontogênico calcificante $(n=4,1,57 \%)$, cisto odontogênico glandular $(n=1,0,39 \%)$, cementoblastoma ( $n=1,0,39 \%)$, fibroma ameloblástico $(n=1,0,39 \%)$, mixoma odontogênico $(n=1,0,39 \%)$, tumor odontogênico adenomatóide $(n=2,1 \%)$, tumor odontogênico epitelial calcificante ( $\mathrm{n}=1,0,39 \%)$, e carcinoma ameloblástico ( $\mathrm{n}=1,0,39 \%)$.

Quanto ao sexo dos pacientes, o número de casos foi ligeiramente maior em mulheres (130:125) com uma idade média de 34 anos $(\mathrm{DP}= \pm 20,53)$. A distribuição de acordo com a localização anatômica dos casos resultou em 137 casos (53,7\%) na mandíbula, 81 (31,8\%) na maxila e 37 (14,5\%) em que a localização não foi relatada nos laudos. 
Tabela 1: Diagnóstico histopatológico, sexo e localização anatômica das lesões odontogênicas avaliadas neste estudo.

\begin{tabular}{|c|c|c|c|c|c|c|}
\hline \multirow{2}{*}{$\begin{array}{l}\text { Diagnóstico } \\
\text { Cistos odontogênicos }\end{array}$} & \multirow[t]{2}{*}{$\mathbf{n}$} & \multicolumn{2}{|c|}{ Sexo } & \multicolumn{3}{|c|}{ Localização } \\
\hline & & Fem. & Masc. & Max. & Mand. & N.I. \\
\hline Cisto radicular & 83 & 41 & 42 & 37 & 37 & 9 \\
\hline Cisto dentígero & 81 & 39 & 42 & 23 & 45 & 13 \\
\hline Ceratocisto odontogênico & 28 & 12 & 16 & 8 & 18 & 2 \\
\hline Cisto odontogênico calcificante & 4 & 3 & 1 & 1 & 1 & 2 \\
\hline Cisto odontogênico glandular & 1 & 0 & 1 & 0 & 1 & o \\
\hline Total COs & 197 & 95 & 102 & 69 & 102 & 26 \\
\hline \multicolumn{7}{|l|}{ Tumores odontogênicos } \\
\hline Odontoma & 26 & 16 & 10 & 8 & 11 & 7 \\
\hline Ameloblastoma & 25 & 15 & 10 & 4 & 18 & 3 \\
\hline $\begin{array}{l}\text { Tumor odontogênico } \\
\text { adenomatóide }\end{array}$ & 2 & 1 & 1 & 0 & 2 & o \\
\hline Mixoma odontogênico & 1 & 1 & 0 & 0 & 1 & O \\
\hline Fibroma ameloblástico & 1 & o & 1 & o & 1 & o \\
\hline Cementoblastoma & 1 & 1 & 0 & 0 & 1 & 0 \\
\hline $\begin{array}{l}\text { Tumor odontogênico epitelial } \\
\text { calcificante }\end{array}$ & 1 & 0 & 1 & 0 & O & 1 \\
\hline Carcinoma ameloblástico & 1 & 1 & o & o & 1 & o \\
\hline Total TOs & 58 & 35 & 23 & 12 & 35 & 11 \\
\hline Total LOs & 255 & 130 & 125 & 81 & 137 & 37 \\
\hline
\end{tabular}

Legenda: $\mathrm{n}=$ Número total de casos; Fem. = Feminino; Masc. = Masculino; Max. = Maxila; Mand. = Mandíbula; N.I. = Não informado; COs = Cistos Odontogênicos; TOs = Tumores Odontogênicos; LOs = Lesões Odontogênicas.

\section{Dıscussão}

Os COs e os TOs representam um amplo e heterogêneo grupo de lesões maxilofaciais, fato que justifica a importância de estudos que definam a prevalência e as principais características clínico-patológicas destas lesões na população ${ }^{18}$. As discussões acerca da diferenciação entre LOs císticas e neoplásicas têm resultado em constantes reclassificações sugeridas pela OMS, com destaque ao ceratocisto odontogênico e cisto odontogênico calcificante, considerados tumores em 2005 e cistos em $2017^{14,17}$. Desse modo, o enquadramento dessas lesões em grupos específicos está relacionado, principalmente, com a sua etiopatogenia e o seu comportamento biológico.

Neste estudo, foram identificados um total de 255 LOs diagnosticadas em um hospital no sul do Brasil, sendo que destas 197 eram COs. Logo, nossos resultados mostraram uma 
maior prevalência de COs quando comparados aos TOs, assim como nos estudos de Al-Rawi et al. ${ }^{1}$, Farias et al..$^{19} \mathrm{e}$ Johnson et al..$^{20}$. No que se refere ao sexo dos pacientes, nossos resultados demonstraram que o número de casos foi ligeiramente maior em mulheres e, apesar de alguns estudos apresentarem uma maior predileção pelo sexo masculino $0^{8,9}$, isso pode refletir a maior procura por serviços de saúde pelas mulheres ${ }^{19,21}$. Além disso, a predileção por sexo em LOs é um parâmetro bastante variável em diferentes estudos ${ }^{1,8,9,22}$.

Com relação à idade, houve uma ampla variação de acordo com o diagnóstico das lesões, com uma média de 34 anos $(\mathrm{DP}= \pm 20,53)$. Considerando as lesões de forma individualizada, observamos que as médias apresentaram uma alta variação entre segunda e oitava décadas de vida. Na literatura, as informações também são amplas quando referentes às faixas etárias para ocorrência de LOs. Nos estudos de Al-Rawi et al. ${ }^{1}$ e Baghaei et al. ${ }^{4}$, houve uma maior prevalência de COs e TOs em pacientes na segunda, terceira e quarta décadas de vida. Considerando apenas TOs, Nalabolu et al. ${ }^{23}$ descreveram que as incidências de pico das lesões foram observadas nas terceiras e quartas décadas de vida. Já no estudo de Osterne et al. ${ }^{16}$, os TOs ocorreram em uma ampla faixa de idades, variando de 1 a 78 anos, com idade média de 30,5 anos. Estas variações podem estar relacionadas com a alta variedade de lesões que fazem parte do grupo das LOs. Quanto à localização anatômica das LOs neste estudo, a mandíbula foi o local de ocorrência mais comum, totalizando $54 \%$ dos casos. Outros estudos demonstram que a mandíbula é a localização mais prevalente em casos COs e $\operatorname{TOs}^{18,23,24}$. Dentre os laudos analisados neste estudo, foi observado que 14,5\% não continham informações exatas acerca da localização anatômica das lesões avaliadas. Embora este seja um fator de limitação no presente trabalho, o mesmo serve para reforçar a importância de solicitar o completo preenchimento das fichas clínicas dos pacientes. As informações presentes nos laudos são essenciais para avaliação dos casos estudados e para o enriquecimento de possíveis pesquisas futuras.

No presente estudo, cistos radiculares e dentígeros foram responsáveis por $64 \%$ dos casos, seguido por ceratocisto odontogênico (11\%). Esses três tipos de cistos odontogênicos têm sido descritos como os cistos mais prevalentes em diferentes estudos ${ }^{22,25,26}$. De forma semelhante aos nossos resultados, Mendez et al. ${ }^{21}$ demonstraram que as lesões inflamatórias periapicais foram as mais comuns dentre os espécimes analisados em um laboratório de patologia oral. Estes resultados demonstram que dentre as lesões císticas, independentemente de ser um laboratório de patologia oral ou de patologia médica em um serviço hospitalar, o cisto radicular aponta como o mais prevalente.

Neste estudo, os TOs totalizaram cerca de $23 \%$ do total de casos de LOs diagnosticados no serviço. Observou-se, também, uma maior prevalência de TOs benignos em relação aos malignos. Dentre os tipos benignos, os mais prevalentes foram ameloblastoma e odontoma, representando uma taxa de cerca de $10 \%$ cada nesta amostra. Outros TOs, incluindo cementoblastoma, fibroma ameloblástico, tumor odontogênico adenomatóide e o caso maligno de carcinoma ameloblástico, compreenderam somente cerca de 3\% do total da amostra. Diferentes estudos demonstram uma baixa incidência de TOs, sendo que os tipos histológicos normalmente mais prevalentes são o odontoma e o ameloblastoma ${ }^{11,14,18,24,27}$, corroborando com os nossos achados. Os TOs malignos representaram 0,39\% dentre todas as LOs avaliadas no presente estudo. Apenas um caso de carcinoma ameloblástico foi observado. As neoplasias odontogênicas malignas são entidades $\operatorname{raras}^{19,21,28}$ e diferentes estudos também mostram a baixa prevalência destas lesões ${ }^{24,29}$. De acordo com a classificação mais recente da $\mathrm{OMS}^{17}$, as lesões classificadas como neoplasias malignas de origem odontogênica são carcinoma ameloblástico, carcinoma intraósseo primário, carcinoma odontogênico esclerosante, carcinoma odontogênico de células claras, carcinoma odontogênico de células fantasmas, carcinossarcoma odontogênico e sarcomas odontogênicos. Apesar de sua frequência extremamente baixa, os TOs malignos são causa importante de extensos procedimentos cirúrgicos na região oral e maxilofacial, ocasionando importante morbidade aos pacientes ${ }^{30}$. 
É importante mencionar que a análise do presente estudo foi realizada em um hospital terciário referência em oncologia. No geral, entende-se que em ambientes hospitalares o encaminhamento de pacientes com tumores na região de cabeça e pescoço é potencialmente mais frequente quando comparado a outros serviços. Nesse contexto, a semelhante prevalência entre ameloblastomas e odontomas chama a atenção neste estudo, considerando que os odontomas podem ser considerados hamartomas e, normalmente, são mais prevalentes em serviços específicos de patologia bucal. Entretanto, conforme já mencionado, estes são os tipos normalmente mais prevalentes dentre os TOs e estes resultados refletem a realidade do que é normalmente observado em amostras de LOs.

\section{CONCLUSÃO}

Neste estudo, as LOs mais frequentes por tipos histopatológicos foram cisto radicular, cisto dentígero, ceratocisto odontogênico, odontoma e ameloblastoma. Os dados apresentados no presente trabalho corroboram com a literatura no que se refere à prevalência e à raridade do diagnóstico de TOs. Pesquisas avaliando as características clínicas e as frequências de LOs permitem uma definição mais precisa da sua ocorrência em populações específicas e em diferentes serviços, possibilitando a identificação das características demográficas mais comuns associadas ao seu desenvolvimento.

\section{CONFLITO DE INTERESSES}

Os autores declaram não haver conflito de interesses.

\section{REFERÊNCIAS}

1. Al-Rawi NH, Awad M, Al-Zuebi IE, Hariri RA, Salah EW. Prevalence of odontogenic cysts and tumors among UAE population. J Orofac Sci. 2013 Jul;5(2):95-9.

2. Pereira JV, Figueiredo DU, Souza EA, Holmes TSV, Gomes DQC, Cavalcanti AL. Prevalência de cistos e tumores odontogênicos em pacientes atendidos na Fundação Assistencial da Paraíba: estudo retrospectivo. Arquivos em Odontologia. 2010 Abr-Jun;49(2):75-81.

3. Urrutia SN, Figueiredo R, Escoda CG. Retrospective clinicopathological study of 418 odontogenic cysts. Med Oral Patol Cir Bucal. 2010 Set;15(5):767-73.

4. Baghaei F, Zargaran M, Najmi H, Moghimbeigi A. A clinicopathological study of odontogenic cysts and tumors in Hamadan, Iran. J Dent Univ Med Sci. 2014 Dez;15(4):167-72.

5. Imran A, Jayanthi P, Gobu SC. Classification of odontogenic cysts and tumors - Antecedents. J Oral Maxilofac Pathol. 2016 Maio-Ago;20(2):269-71

6. Arce K, StreffCS, Ettinger KS. Pediatric odontogenic cysts of the jaws. Oral Maxilofacial Surg Clin N Am. 2016;28:21-30.

7. Sharifian M], Khalili M. Odontogenic cysts: a retrospective study of 1227 cases in an Iranian population from 1987 to 2007. J Oral Sci. 2011; 53(3):361-7.

8. Costa DOP, Maurício AS, Faria PAS, Silva LE, Taylor AM, Lourenço SQC. Odontogenic tumors: a retrospective study of four Brazilian diagnostic pathology centers. Med Oral Patol Oral Cir Bucal. 2012 Maio;17(3):389-94.

9. AlSheddi MA, AlSenani MA, AlDosarib AW. Odontogenic tumors: analysis of 188 cases from Saudi Arabia. Ann Saudi Med. 2015;35(2):146-50.

10. Deepthi P, Beena V, Padmakumar S, Rajeev R, Sivakumar R. A study of 1177 odontogenic lesions in a South Kerala population. J Oral Maxillofac Pathol. 2016 Maio-Ago;20(2):202.

11. Iyogun CA, Omitola OG, Ukegheson GE. Odontogenic tumors in Port Harcourt: South-South geopolitical zone of Nigeria. J Oral Maxillofac Pathol. 2016 Maio-Ago;20(2):190-3. 
12. Sekerci AE, Nazlım S, Etoz M, Denız K, Yasa Y. Odontogenic tumors: a collaborative study of 218 cases diagnosed over 12 years and comprehensive review of the literature. Med Oral Patol Oral Cir Bucal. 2015 Jan;20(1):34-44.

13. Goteti SHL. Odontogenic tumors: a review of 675 cases in Eastern Libya. Niger ] Surg. 2016 Jan-Jun;22(1):37-40.

14. Bianco BCF, Sperandio FF, Hanemann JAC, Pereira AAC. New WHO odontogenic tumor classification: impact on prevalence in a population. J Appl Oral Sci. 2019 Nov;28:e20190067.

15. Barnes L, Eveson JW, Reichart P, Sidransky D. World Health Organization Classification of tumours. Pathology and genetics of head and neck tumours. Lyon: IARC PRESS; 2005.

16. Osterne RLV, Brito RGM, Alves APNN, Cavalcante RB, Sousa FB. Odontogenic tumors: a 5-year retrospective study in a Brazilian population and analysis of 3406 cases reported in the literature. Oral Surg Oral Med Oral Pathol Oral Radiol Endod. 2011;111:474-81.

17. Wright JM, Vered M. Update from the 4th Edition of the World Health Organization Classification of Head and Neck Tumours: Odontogenic and Maxillofacial Bone Tumors. Head and Neck Pathol. 2017;11:68-77.

18. Raj A, Ramesh G, Nagarajappa R, Pandey A, Raj A. Prevalence of odontogenic lesions among the Kanpur population: an institutional study. J Exp Ther Oncol. 2017;12(1):35-42.

19. Farias JG, Souza RCA, Hassam SF, Cardoso JA, Ramos TCF, Santos HKA. Epidemiological study of intraosseous lesions of the stomatognathic or maxillomandibular complex diagnosed by a Reference Centre in Brazil from 2006-2017. Br] Oral Maxillofac Surg. 2019 Sep;57(7):632-7.

20. Johnson NR, Savage NW, Kazoullis S, Batstone MD. A prospective epidemiological study for odontogenic and non-odontogenic lesions of the maxilla and mandible in Queensland. Oral Surg Oral Med Oral Pathol Oral Radiol. 2013;115(4):515-22.

21. Mendez M, Carrard VC, Haas AN, Lauxen Ida S, Barbachan J], Rados PV, et al. A 10-year study of specimens submitted to oral pathology laboratory analysis: lesion occurrence and demographic features. Braz Oral Res. 2012 Jun;26(3) 235-41.

22. Tamiolakis P, Thermos G, Tosios KI, Sklavounou-Andrikopoulou A. demographic and clinical characteristics of 5294 jaw cysts: a retrospective study of 38 years. Head Neck Pathol. 2019:13(4):587-96.

23. Nalabolu GRK, Mohiddin A, Hiremath SKS, Manyam R, Bharath TS, Raju PR. Epidemiological study of odontogenic tumours: an institutional experience. J Infect Public Health. 2016;10(3):324-30.

24. Silva LP, Serpa MS, Sobral APV, Arruda JAA, Silva LVO, Noronha MS, et al. A retrospective multicentre study of cystic lesions and odontogenic tumours in older people. Gerodontology. 2018 Dez;35(4):325-32.

25. Soluk-Tekkeşin M, Wright]M. The World Health Organization Classification of Odontogenic Lesions: a Summary of the Changes of the 2017 (4th) Edition. Turk Patoloji Derg. 2018;34(1):1-18.

26. Rioux-Forker D, Deziel AC, Williams LS1, Muzaffar AR. Odontogenic cysts and tumors. Ann Plast Surg. 2019;82(4):46977 .

27. Bhat A, Mitra S, Chandrashekar C, Solomon M, Kulkarni S. Odontogenic cysts and odontogenic tumors in a large rural area from India. A 10-year reflection. Med Pharm Rep. 2019;92(4):408-12.

28. Ahire MS, Tupkari JV, Chettiankandy T], Thakur A, Agrawal RR. Odontogenic tumors: a 35-year retrospective study of 250 cases in an Indian (Maharashtra) teaching institute. Indian ] Cancer. 2018 Jul-Sep;55(3):265-72.

29. Lei F, Chen JY, Wang WC, Lin LM, Huang HC, Ho KY, et al. Retrospective study of oral and maxillofacial lesions in older Taiwanese patients. Gerodontology. 2015 Dec;32(4):281-7.

30. Mosqueda Taylor A, Meneses García A, Ruíz Godoy Rivera LM, Suárez Roa Mde L, Luna Ortiz K. Malignant odontogenic tumors. A retrospective and collaborative study of seven cases. Med Oral. 2003 Mar-Apr;8(2):110-21. 